\title{
A STABILITY CRITERION FOR QUASI-HARMONIC VIBRATIONS*
}

\author{
BY AUREL WINTNLR (The John Hopkins University)
}

1. Let $\omega=\omega(t)$ be a continuous function which is periodic, choose its period to be $\pi$, and call

$$
x^{\prime \prime}+\omega^{2}(t) x=0
$$

a stable oscillator if all solutions $x(t)$ (i.e., if two, linearly independent solutions) stay bounded as $|t| \rightarrow \infty$. It is understood that $\omega(t)$ is assumed to be real-valued, which means that $\omega^{2}(t) \geqq 0$. Here the sign of equality is allowed (for certain $t$-values, which can be isolated or form intervals), but it will be assumed that $\omega(t)$ does not vanish identically, i.e., that the case $x \equiv 0$ of (1) is excluded.

According to Liapounoff [1], (1) must be stable if

$$
\int_{0}^{\pi} \omega^{2}(t) d t<4 / \pi \text {. }
$$

But it is also known [2] that if $4 / \pi$ is replaced by any numerical constant greater than $4 / \pi$, then the assumption which thus results from (2) will allow the instability of (1) (for certain frequency functions $\omega(t)$, to be chosen in an appropriate manner). In other words, $4 / \pi$ is the best absolute constant in (2).

This is not, of course, at variance with the fact that if $\omega(t)$ is the constant 1 , then (1) is stable but (2) is violated (since $\pi>4 / \pi$ ). But it can be expected that the absolute constant $4 / \pi$ will be improved if it is assumed that $\omega(t)$ keeps away from 0 by a fixed amount. In this regard, a result of P. S. Gusarova [3] states that if

$$
\omega(t) \geqq 1 \text {, }
$$

then

$$
\int_{0}^{\pi} \omega^{2}(t) d t \leqq 12 / \pi,
$$

a relaxation of (2), will guarantee the stability of (1).

2. This appears to clear up the situation in a satisfactory manner. Actually, the stability eriterion (3)-(4) is unsatisfactory from the point of view of most applications. For, from such a point of view, the stability of (1) ought to be ensured by imposing a limitation on a characteristic which measures the relative degree of the non-constancy of $\omega(t)$, rather than on a characteristic measuring the amount by which $\omega(t)$ keeps away from 0 . But the criterion (3)-(4) is of this latter kind.

Needless to say, the criterion of the desired type will also have to involve a limitation of the mean-value of $\omega^{2}(t)$ over a period; see (2) or (4). In the desideratum, the latter limitation, supplied by the bounds $4 / \pi, 12 / \pi$ in the respective criteria (2), (3)-(4) of Sec. 1 , will have to be expressed in terms of the characteristic measuring the non-constancy of $\omega(t)$.

3. The simplest choice of such a characteristic is the quotient

$$
\vartheta=(M-m) /(M+m),
$$

*Received November 5, 1957. 
where

$$
m=\min _{0 \leq t \leq \pi} \omega^{2}(t), \quad M=\max _{0 \leq t \leq \pi} \omega^{2}(t)
$$

hence

$$
0 \leqq m \leqq M<\infty \text { and } M>0
$$

(in fact, if $M=0$, then (1) reduces to the unstable, but trivial, equation $x^{\prime \prime}=0$, excluded since the beginning of Sec. 1). It is clear from (6) and (7) that the quotient (5) exists and is subject to the inequalities

$$
0 \leqq \vartheta<1
$$

and that (1) is the closer to the state of a harmonic oscillator $(\vartheta=0, \omega(t)=$ const. $>0)$ the closer is $\vartheta$ to 0 . For this reason, the following criterion, the deduction of which is the purpose of this note, is along the lines of the desideratum formulated in Sec. 2:

If the coefficient function of an oscillator (1) does not vanish identically and is of period $\pi$, then all solutions $x(t)$ of (1) stay bounded, as $|t| \rightarrow \infty$, whenever the mean-value of the squared "instantaneous frequency" $\omega(t)$ satisfies the inequality

$$
\frac{1}{\pi} \int_{0}^{\pi} \omega^{2}(t) d t<Q\left(1-\vartheta^{2}\right)^{1 / 2}
$$

where $\vartheta$ denotes the characteristic of $\omega(t)$ defined by (5) and (6), and $Q$ is a certain numerical constant,

$$
Q=0.8043 \cdots
$$

It will remain undecided whether (10) is the best absolute constant allowed in this stability criterion. But it is seen from (10) that the value of the product $\pi Q$ certainly exceeds the value of $4 / \pi$. Hence, if $\vartheta$ is reasonably small, then the criterion (9) is more favorable than Liapounoff's criterion (2).

Note that, the quotient (5) being a relative ("percentile") characteristic of $\omega(t)$, nothing like an absolute limitation (3) is involved in (9).

4. The sufficiency of (9) for the stability of (1) will be concluded from the following criterion, proved by Borg [4] as a counterpart of Liapounoff's criterion (quoted at the beginning of Sec. 1): If $f(t)$ is a real-valued, continuous function of period $T$, and if $f(t)$ is non-negative throughout but does not vanish identically, then all solutions $x(t)$ of

$$
x^{\prime \prime}+f(t) x=0
$$

will stay bounded (as $|t| \rightarrow \infty$ ) whenever the coefficient of (11) is restricted by the condition

$$
\int_{0}^{T} f(t) d t<64 P^{4} / 3 T^{3}
$$

where

$$
P=\int_{0}^{1 / 2 \pi}\left(1+\sin ^{2} \varphi\right)^{-1 / 2} d \varphi .
$$

In order to identify (11) with (1), let $f(t)=\omega^{2}(t)$ and $T=\pi$. Then (12) can be written in the form 


$$
\frac{1}{\pi} \int_{0}^{\pi} \omega^{4}(t) d t<64 P^{4} / 3 \pi^{4} .
$$

Consequently., it is sufficient to show that (9) is implied by (14).

5. First, if $f_{1}(t)$ and $f_{2}(t)$, where $u \leqq t \leqq v$, are arbitrary continuous functions satisfying the inequalities

$$
0<m_{i} \leqq f_{i}(t) \leqq M_{i}, \text { where } i=1,2,
$$

then, by "the (partial) converse of Schwarz's inequality" [5], the quotient

$$
4 \int_{u}^{0} f_{1}^{2}(t) d t \cdot \int_{u}^{0} f_{2}^{2}(t) d t /\left[\int_{u}^{0} f_{1}(t) f_{2}(t) d t\right]^{2}
$$

cannot exceed the square of

$$
\left(M_{1} M_{2} / m_{1} m_{2}\right)^{1 / 2}+\left(m_{1} m_{2} / M_{1} M_{2}\right)^{1 / 2} .
$$

If this is applied to

$$
f_{1}(t)=\omega^{2}(t), \quad f_{2}(t)=1 \quad \text { and } \quad u=0, v=\pi,
$$

it is seen from (6) that

$$
4 \pi \int_{0}^{\pi} \omega^{4}(t) d t \leqq\left[(m / M)^{1 / 2}+(M / m)^{1 / 2}\right]^{2}\left[\int_{0}^{\pi} \omega^{2}(t) d t\right]^{2} .
$$

Consequently, (14) is certainly satisfied if

$$
\frac{1}{\pi} \int_{0}^{\pi} \omega^{2}(t) d t<\frac{16 P^{2} / \pi^{2}}{3^{1 / 2}\left[(m / M)^{1 / 2}+(M / m)^{1 / 2}\right]} .
$$

In view of the end of Sec. 4, this means that the proof will be complete if it is shown that (9) is implied by (15).

6. It is readily verified from (5) that

$$
(m / M)^{1 / 2}+(M / m)^{1 / 2}=2 /\left(1-\vartheta^{2}\right)^{1 / 2} .
$$

Hence (15) can be written in the form (9) if $Q$ is defined by

$$
Q=2^{3} \cdot 3^{-1 / 2} P^{2} / \pi^{2} .
$$

Finally, the substitution $\alpha=\sin \varphi$ transforms (13) into

$$
P=\int_{0}^{1}\left(1-\alpha^{4}\right)^{-1 / 2} d \alpha .
$$

But (17) is the celebrated constant of Stirling [6], the complete lemmiscatic integral (of first kind). Since

$$
P=1.3110 \cdots,
$$

(10) follows from (16).

7. As an illustration, consider Mathieu's equation

$$
x^{\prime \prime}+(a+b \cos 2 t) x=0,
$$


where $a, b$ are constants. This is an equation of the form (1), with a positive $\omega^{2}(t)$ of period $\pi$ and of mean-value $a$, if $0<b<a$. Since (6) reduces to $m=a-b$ and $M=$ $a+b$ in this case, it is seen from (5) that

$$
1-\vartheta^{2}=4 c /(1+c)^{2}, \text { where } c=b / a \text {. }
$$

Hence, condition (9) becomes $a<Q c^{1 / 2} /(1+c)$.

Since $c=b / a$, the latter inequality can be written in the form

$$
a / b<4 Q^{2} /(a+b)^{2} \text {, where } 0<b<a \text {. }
$$

Consequently, Mathieu's equation (19) is stable (and, what is more, "disconjugate") whenever its parameters $a, b$ satisfy the conditions (20), where $Q$ is the value (10), defined by (16) and (17).

\title{
REFERENCES
}

1. See E. R. Forsyth, Theory of differential equations, vol. IV, part III, pp. 425-431, 1902

2. E. R. van Kampen and A. Wintner, Am. J. Math. 59, 270-274 (1937)

3. See J. L. Geronimus, A. M. Ljapunow, Berlin, p. 80, 1954

4. G. Borg, Arkiv för Mat., Astr. och Fys. 31A, no. 1, 29 (1944)

5. See G. P6lya and G. Szegö, Aufgaben und Lehrsatze aus der Analysis, chap. II, no. 93, 1925

6. See C. F. Gauss, Werke, vol. XI , p. 145, pp. 542-543

\section{A HEAT CONDUCTION PROBLEM INVOLVING A SPECIFIED MOVING BOUNDARY*}

\author{
By R. E. GIBSON (Imperial College, London)
}

1. Introduction. Physical problems governed by an equation of heat conduction type occasionally arise which require a condition to be satisfied on a boundary moving at a specified rate. In the problems with which we are concerned the movement of the boundary is generated by the accretion there of new material and not by deformation of the medium. Typical examples are: the dissipation of excess pore water pressure in a clay layer upon which fresh material is being deposited [1], and the type of thermal problem considered by Benfield [2] relating to the theory of the formation of the earth on the dust cloud hypothesis. The moving boundary is also a feature of thermal problems where a change in state of the conducting medium occurs [3]. Here, the motion of the boundary separating the phases is not given and must be determined as part of the solution. We can hope, therefore, that our problems, where the motion of the boundary is given, will prove to be less intransigent.

While working on one-dimensional problems of this type, we found that exact solutions could be obtained in two cases, namely, when the rate of movement of the boundary is proportional to $t^{-1 / 2}$ and when it is constant. Although in few actual problems may these conditions be realized, we believe our results to be of interest since it appears unlikely that closed solutions can be obtained for an arbitrary motion of the boundary.

2. Problem with spherical symmetry. We consider first a case with spherical

*Received October 22, 1957. 